\title{
Nuclear Power from Lunar ISRU
}

\author{
Peter J Schubert* \\ IUPUI, Indianapolis, IN 46202, USA
}

Submission: August 07, 2019; Published: August 29, 2019

*Corresponding author: Peter J Schubert, IUPUI, Indianapolis, IN 46202, USA

\begin{abstract}
Thorium on the lunar surface can be transmuted into fissile uranium suitable for a controlled chain reaction to provide heat. Thorium is fertile, requiring bombardment by neutrons to become a suitable nuclear fuel. Oxides of thorium are dense and can be concentrated and beneficiated from comminuted regolith via inertial or thermal means. A neutron flux can be provided by encasing thoria within a beryllium and graphite vessel, which emits neutrons upon exposure to gamma rays or galactic cosmic rays. After a brief period at protactinium the transmuted material becomes U-233, a desirable fuel because decay product half-lives are below 100 years. When compressed into fuel pellets the uranium oxide is configured into a reactor through which a working fluid can extract thermal power. With regolith tailings as shielding such a reactor can operate safely for 30 years. A century later, the site can be harvested for specialty elements and then made available for other uses. The advent of launch-safe nuclear rockets in space greatly expands the potential for in situ resource utilization, a space-based economy, and profitable exploitation of the asteroid belt.
\end{abstract}

\section{Introduction}

Lunar missions involving in situ resource utilization (ISRU) require ample supplies of thermal and electrical energy. Solar power is a poor choice, being diffuse, and intermittent. Operations in permanently shadowed regions (PSR) get no sunlight, inviting complex reflection or conversion and beaming schemes to power operations on the floors of ultra-cold craters. Nuclear power is an oft-cited option, as technology is readily available for the reactor to only go "hot" after being installed on the moon. Even for such reactor designs, the uranium needed is already part of the payload, and this material is radioactive, albeit at a low level. Many people, often unaware of radioactive uranium in their own granite countertops, have a deep-seated fear of anything radioactive inside a rocket, worried it might explode and spread the contamination across the environment. It is possible, therefore, that even "nearly safe" nuclear reactors launched from earth will experience significant public protest, resistance, Congressional pressure, and lawsuits. Another solution exists and is explored in this paper.

The US nuclear power development, starting with the Manhattan Project, had two primary purposes: electric power generation, and radioisotope generation for bombs made of uranium, plutonium, and hydrogen. Because of this dual focus, the enrichment of the U-235 fraction of uranium was the pathway used for nearly all applications. A modest effort in breeder reactors was made, which pursued the transmutation of thorium via the neutron flux from $\mathrm{U}-235$ reactors to make $\mathrm{U}-233$. This lighter isotope of uranium is fissile, as is $\mathrm{U}-235$ (most uranium is U-238, which is stable), and makes a suitable fuel, but less wellsuited to bomb-making. Such work is all but extinct in 2019, and is almost forgotten, except by a few passionate advocates. The moon has very little uranium but amounts of thorium which are at or above typical abundances on earth's crust - from which the moon is believed to have been formed. If this resource can be concentrated and transmuted, it will be possible to fuel nuclear reactors which can be launched completely free of radioactive material. Furthermore, the high-density nuclear fuel comes from local resources on the moon, helping to reduce launch mass of each such reactor.

\section{Materials and Methods}

\section{Concentrating Thorium}

Thorium is found across a large expanse of the moon's Near Side with concentrations of 10-20 parts per million (ppm) [1]. Impact fracturing from meteoric bombardment has embedded or aggregated thorium dioxide (ThO2, or "thoria") into other minerals, classified as agglutinates [2]. Thoria is an especially dense mineral with a specific gravity 10 times that of water. A straightforward concentration method is to comminute thorium-rich surface dust (average diameter 70 microns $[3,4]$ ) by grinding or milling to nanometer-sized particulates and then separate them in the lunar gravity using standard sorting techniques. Lunar soil, called regolith, includes free particles of iron-nickel metal left by impacts of stony iron type meteorites. 
Native alloys of these metals will have densities of approximately 8 and may not segregate effectively from thoria-rich agglutinates. Being magnetic, the iron-nickel fraction can be extracted by electromagnet, perhaps driven by electric current delivered by arrays of solar cells. This is a simple and low-energy method of concentrating thorium, likely to be used first.

To achieve greater purity and higher concentration of thorium another method is to exploit the exceptionally high melting point of thoria mineral, at around $3500 \mathrm{~K}$. In addition to its value as a nuclear fuel, this refractory property of thoria has great value in other areas of lunar ISRU, including the extraction of oxygen and silicon from regolith [5,6]. At the extreme temperature needed to refine thoria, crucible materials are a challenge. The skull crucible method7 was developed to produce crystals of refractory zirconia and uses radio frequency (rf) inductive heating to melt the interior of a bolus or "gob" of mineral. The rf coils and the exterior of the bolus are cooled with a working fluid in communication with a radiative heat exchanger (convection is absent on the moon, and conduction through regolith is impractically slow), such that the interior of the charge is liquefied while the exterior remains solid. The gentle gravity of the moon (1/6 that of earth) will cause thoria to settle to the bottom of the melt. The supernatant magma is then poured off, perhaps into forms to create bricks for paving and building, and the remainder will be concentrated thoria. A fortuitous side benefit of the skull crucible method is the evolution of abundant amounts of oxygen, released from common lunar minerals such as quartz, olivine, anorthosite, pyroxene, and ilmenite [7-10]. The skull crucible method is obviously energy intensive, and is therefore a second generation thoria concentration method, used once the first U-233 fission reactor is operating on the moon.

\section{Thorium Transmutation}

Neutron bombardment of thorium (element 90, mass 232) results in its transmutation to protactinium ( $\mathrm{Pa}$, element 91). The extra neutron, being composed of a proton plus an electron, decays by electron emission (beta radiation) to leave behind an additional proton and thereby change the chemical identification of this isotope. The Pa-233 intermediate product has a half-life of 27 days and decays by beta radiation to become U-233. This isotope of uranium is fissile and suitable as a nuclear fuel. ${ }_{90}^{232} \mathrm{Th}+n \rightarrow{ }_{90}^{232} \mathrm{Th}+\beta^{-} \rightarrow{ }_{91}^{231} \mathrm{Th}+\beta^{-} \stackrel{27 \text { days }}{\longrightarrow}{ }_{92}^{233} \mathrm{U}$

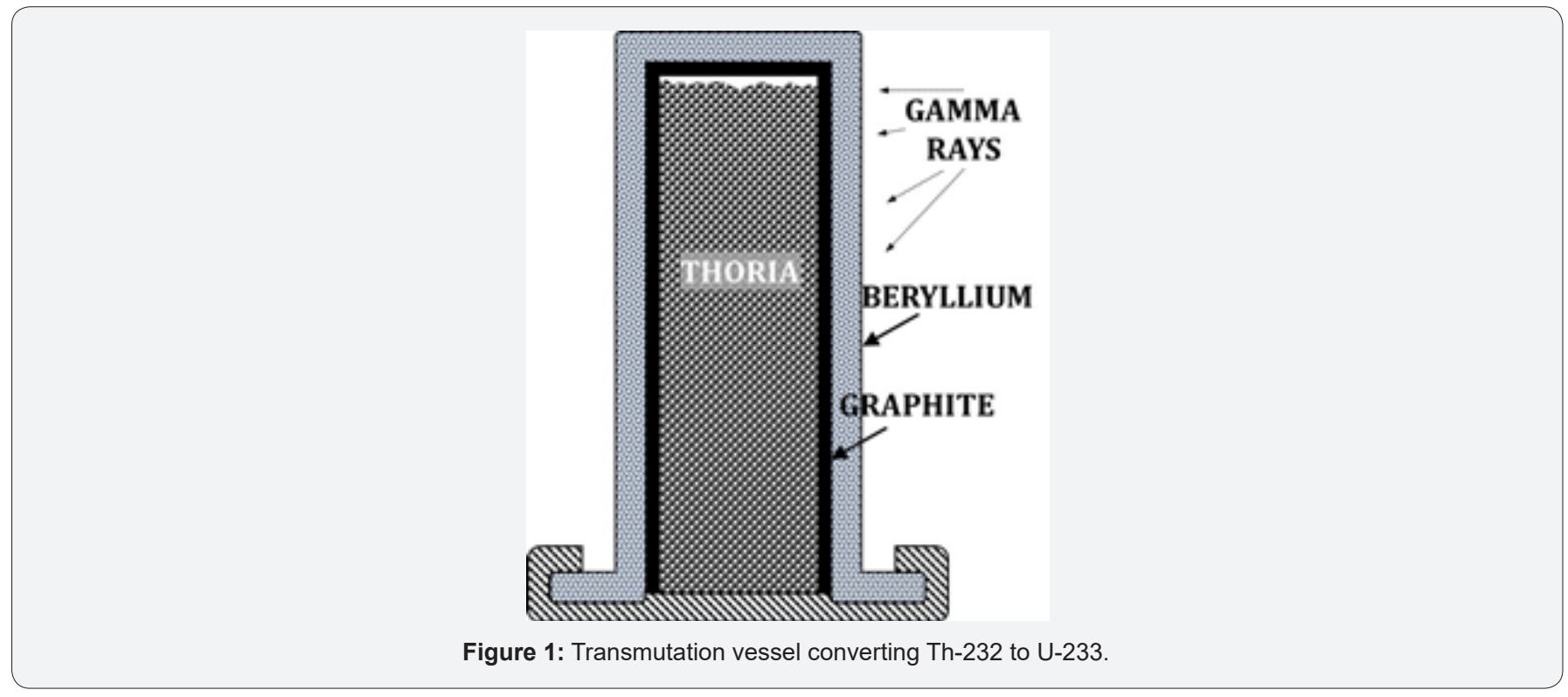

Although space is filled with radiation, very little is neutrons. Outside of an atomic nucleus, wild neutrons have a half-life of 10.3 minutes, only slightly longer than the transit time of light from the sun to the earth's orbit. Energetic solar flares produce neutrons in the sun's corona, which, at relativistic speeds, have a retarded decay time relative to the moon, and can reach the surface. However, such neutrons fluxes are infrequent. Further, because the moon rotates relative to the sun with a synodic period of 27.3 days, only a few locations see the sun more than 50 percent of the time, and these are generally remote from thorium ore bodies. A more reliable source of neutrons for transmutation can be obtained by exposing beryllium (Be) to gamma rays. Gamma rays are abundant in space, making them a health risk for humans, and are generated by galactic cores, solar flares, supernovae, and even lightning on earth. There is a gamma ray background pervading the universe for which no source has been conclusively identified and is called the gamma ray "fog". Gamma rays are more energetic than X-rays, with each photon having energies above 100,000 electron volts $(\mathrm{eV})$. Data from the Fermi Gamma Ray Space Telescope indicates a flux of approximately 0.5 photons per second through each square centimeter of space [11]. When gamma rays impinge 
on beryllium metal, neutrons are generated [12]. A Be vessel containing Th and exposed to gamma rays will therefore transmute or "breed" U-233 fuel (Figure 1).

Gamma ray energies from space span seven orders of magnitude in $\mathrm{eV}$, so some neutrons generated will be fast, and thus more likely to pass through without capture. Graphite acts as a neutron moderator to slow down fast neutrons to become thermal (slower) neutrons with a higher capture cross section by the thorium nucleus. To enhance neutron flux within the Th it is advantageous to use a neutron mirror material, which deflects wild neutrons back into the material to be transmuted. Beryllium is an excellent neutron mirror, as is graphite. Therefore, a vessel wall with exterior made of $\mathrm{Be}$ and interior made of graphite is a good design choice. The vessel must protrude proud from the lunar surface, ideally on a hilltop, and be sized to balance neutron capture probability with neutron flux intensity. Figure 1 illustrates one design configuration of a thorium transmutation vessel.

\section{Fuel Considerations}

Nuclear fission reactors often use pellets or spheres containing oxides of the fissile species. The U-233 urania derived from thoria will already be in the oxide form UO2. The enrichment of U-233 in the mineral charge of the transmutation vessel will depend on the concentration of thoria and the time of exposure. Once removed from the vessel, this fuel can be compressed into pellets using mechanical or hydraulic compression. Cylindrical pellets are loaded into fuel rods and inserted into the reactor core. If lunar-sourced U-233 fuel pellets are used, this allows for a nuclear reactor to be launched from earth having no radioactive materials in the payload manifest. Although the lunar surface is depleted of uranium relative to the earth's crust, it is not negligible3. There may be some U-232 contamination of the thoria, and this complicates the thorium fuel cycle. The skull crucible method is one means for separation of thorium from uranium prior to transmutation, if required depending on system considerations. Chemical methods for Th-U separation have been developed as early as 1949 [13].
Thorium fuel cycle byproducts generally decay within a century, greatly reducing the well-known problems of disposing spent nuclear fuel. Some of these byproducts emit gamma rays. It may be possible to accelerate the breeding of U-233 using the hot waste, once the first fuel cycle of a lunar nuclear reactor is completed. The lunar surface is bombarded by radiation constantly: alpha, beta, gamma, energetic protons, and more. Relative to earth, which is protected by an atmosphere and a magnetosphere, humans on the moon must make protection from radiation second only to protection from the vacuum of outer space. It is a hostile environment and requires much shielding. A commonly envisioned method of radiation protection for humans is a layer of regolith some two meters thick. Applying this logic to spent fuel rods, the expedient of a shallow grave, with markers, signs, and beacons, should be sufficient to prevent harm to humans for a hot century [14-16].

\section{Results}

The thorium atom has a radius of 240 picometers, while oxygen is 60 , so that thorium forms $2 / 3$ of the area of the thoria molecule. Assuming that 50 percent of the impinging gamma rays produce a neutron from the Be outer casing, and assuming that 50 percent of these neutrons are absorbed by a thorium atom (including reflection within the vessel), the number of transmutations with 0.5 gamma ray particles arriving per second, per square $\mathrm{cm}$, is $1.6 \mathrm{E} 13$. Assuming a critical mass of $15 \mathrm{~kg}$ of nuclear fuel to start the reactor, and a time to fuel of three years, accounting for two Pa half-lives in this duration, a cylindrical transmutation vessel of 2 meters height requires an interior radius of $21 \mathrm{~cm}$.

One transmutation vessel holds 0.28 cubic meters of thoria. At $12 \mathrm{ppm}$ concentration of thoria, each charge, sufficient for one nuclear reactor with a 30-year supply, requires considerable excavation and concentration operations. For a compacted regolith density of $2200 \mathrm{~kg} / \mathrm{cu} . \mathrm{m}$ this calls for 51,000 metric tons to be processed. Given the lighter gravity on the moon, this requires the same energy as processing $8,600 \mathrm{MT}$ on earth. As a point of comparison, for a large, mature mining operation on earth, this much mass is moved every 20 minutes (Figure 2).

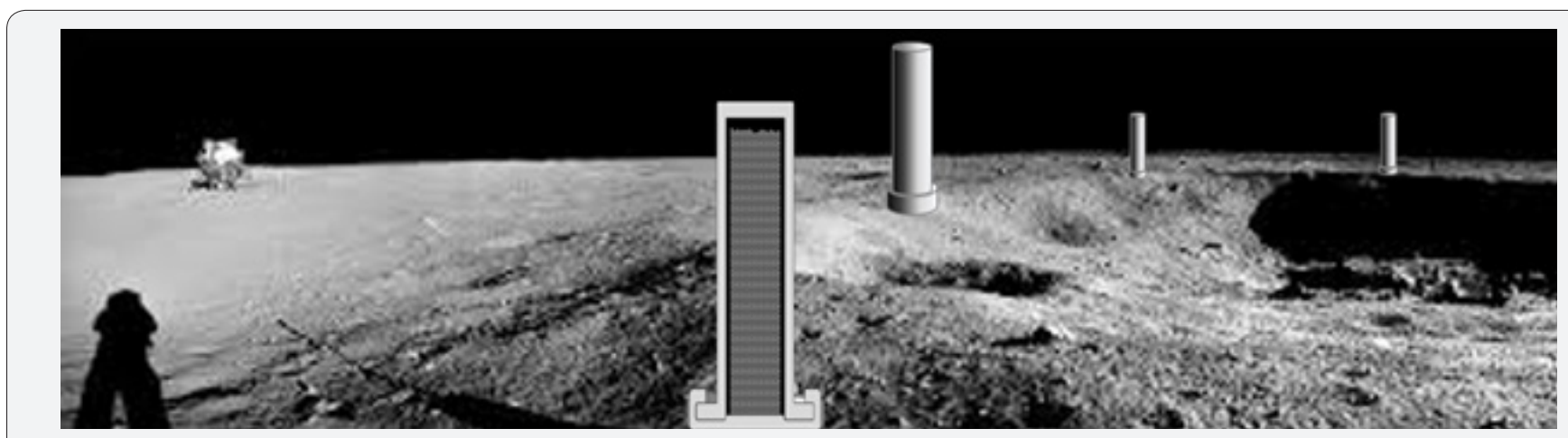

Figure 2: Placement of transmutation vessels along crater rim for gamma ray exposure, foremost cut-away. 


\section{Discussion}

The infrastructure needed to process lunar thorium into nuclear reactor fuel is non-trivial. First generation equipment includes: excavation equipment; ball mills; gravity sorters on a shaker table; electromagnet and solar panels; transmutation vessel; and a fuel pellet compaction device. Second generation equipment includes skull crucible apparatuses driven by $\mathrm{rf}$ generators, and a liquid cooling system with extensive radiative heat exchangers. Three years after the first transmutation vessel is filled, the first lunar nuclear reactor can be started up. Startup generally requires an extra source of neutrons to initiate the fission chain reaction, but as can be seen above, these neutrons can be provided by a layer of beryllium exposed to cosmic rays. Once the first nuclear reactor is operational, second generation refining techniques can be brought online, greatly improving the throughput and purity of the thoria concentration operations. Assuming that mining equipment is operated by battery or fuel cell, the electric power from the nuclear reactor can significantly enhance the rate of ore excavation and concentration. A third generation becomes possible when the first reactor has its fuel rods removed, the gamma rays for which can increase the breeding rate of Th to U-233. It can be seen that the infrastructure investment has an accelerating payback in capability of lunar operations. These favorable economics are further augmented by the additional value which can be provided.

With more power to excavate, the mining operations can provide fuel pellets as an export commodity. Beachhead customers will be other settlements and mining operations on the moon, but also on Mars, and possibly at ore-rich asteroids. To reach these more distant destinations will require fast rockets. The obvious solution is a nuclear thermal rocket (NTR) which superheats hydrogen gas and passes it through a de Laval nozzle to generate thrust. The NTR is powered by U-233, and the hydrogen can be obtained by water ice mining in permanently shadowed regions of the moon. Extraction of water ice from ultra-cold craters can be facilitated by the warmth from spent fuel rods, and a nearby nuclear power station can provide the electrical current needed to electrolyze the water and produce hydrogen. If the NTR-powered spacecraft configure their hydrogen fuel tanks to surround the crew capsule the capture cross section for ionizing space radiation of the fuel significantly reduces exposure for the humans inside.

\section{Conclusion}

Shown here for the first time is a means for safely fueling nuclear reactors in space whereby no radioactive material is exposed to the risks of a rocket launch. Mining operations which are modest by earth standards, are needed to jumpstart an expanding economy of ISRU capabilities which greatly multiply the power, speed, and safety of human operations in space. Nuclear reactors fueled by transmuted thorium native to the moon will drive extraction of hydrogen, oxygen, silicon, aluminum, titanium, and iron. Fast rocket ships powered by NTR open up the entire solar system to extraction of resources useful on earth, such as platinum-rich asteroids which can greatly reduce the cost of fuel cell systems on earth, making possible a hydrogen economy with no atmospheric carbon involved. Nuclear reactors burning U-233 are far safer than reactors currently in use on earth and produce generally short-lived byproducts which decay away in a century. The thorium fuel cycle is ill-suited to bomb makers, so the risks of nuclear war in space, using the technologies presented here, are far less than other means of space warfare. Rather, this new approach has the potential to expand the human economic sphere, open up new frontiers for science and industry, and to help improve life back home on earth.

\section{Acknowledgements}

Helpful discussions were provided by AJ Kragt, Sawyer Powell and Penghui Heng.

\section{Conflict of Interest}

The author declares no conflict of interest.

\section{References}

1. Crawford IA (2015) "Lunar resources." Progress in Physical Geography 39(2): 137-167.

2. Meyer C (2003) The Lunar Petrographic Educational Thin Section Set. Houston, USA.

3. Ferreira E, Dos Santos, Sarango Souza J (2017) Gammas radiation in ceramic capacitors: a study for space missions. Journal of Physics: Conference Series 911: 012004.

4. Heiken G, Vaniman D, French BM (1991) The Lunar Sourcebook: a user's guide to the moon. n.p.: Cambridge; Cambridge University Press, c1991., 1991. IUCAT IUPUI (library catalog), New York, USA.

5. Schubert PJ, Williams J, Bundorf T, Di Sciullo-Jones A (2010) "Advances in Extraction of Oxygen and Silicon from Lunar Regolith," AIAA SPACE 2010, Anaheim, CA, 30 Aug -2 Sept 2010.

6. Schubert P (2011) US 7,935,176, Oxygen Extraction Apparatus and Process.

7. Schubert P (2005) US 6,930,304, Process and Apparatus for Isotope Separation in a Low-Gravity Environment.

8. Schubert P (2006) "Synergistic Construction Mechanisms for Habitats in Space Environs." Int'l. Space Development Conference, Los Angeles, USA.

9. Schubert, Peter J (2004) Isotope separation process and apparatus, therefore.

10. Schubert P (2005) A Novel Method for Element Beneficiation Applied to Solar Panel Production, Space Exploration, Albuquerque, NM.

11. NASA https://fermi.gsfc.nasa.gov/science/eteu/diffuse/ accessed 7 Aug. 2019.

12. Szilard L, Chalmers TA (1934) Detection of Neutrons Liberated from Beryllium by Gamma Rays: a New Technique for Inducing Radioactivity. Nature, 134: 494-495.

13. Bane RW (1949) Separation of Thorium from Uranium. US Patent 2,902: 338-346

14. Cannon R (2018) NASA Robotic Mining Competition.

15. Choi CQ (2010) Moon Map Shows Uranium in Short Supply. Science \& Astronomy.

16. Manutchehr-Danai M (2009) skull crucible. In: Dictionary of Gems and Gemology. 

(C) This work is licensed under Creative
Your next submission with Juniper Publishers will reach you the below assets

- Quality Editorial service

- Swift Peer Review

- Reprints availability

- E-prints Service

- Manuscript Podcast for convenient understanding

- Global attainment for your research

- Manuscript accessibility in different formats

(Pdf, E-pub, Full Text, Audio)

- Unceasing customer service

Track the below URL for one-step submission https://juniperpublishers.com/online-submission.php 